\title{
Corrigendum to "Supervised and Unsupervised Subband Adaptive Denoising Frameworks with Polynomial Threshold Function"
}

\author{
Tierui Gong, ${ }^{1,2}$ Zhijia Yang, $^{1}$ Gengshan Wang, ${ }^{1,2}$ and Ping Jiao ${ }^{1}$ \\ ${ }^{1}$ Department of Industrial Control Networks and Systems, Shenyang Institute of Automation, \\ Chinese Academy of Sciences, Shenyang 110016, China \\ ${ }^{2}$ University of Chinese Academy of Sciences, Beijing 100049, China
}

Correspondence should be addressed to Tierui Gong; gongtierui@sia.cn

Received 16 August 2017; Accepted 13 September 2017; Published 24 September 2017

Copyright (C) 2017 Tierui Gong et al. This is an open access article distributed under the Creative Commons Attribution License, which permits unrestricted use, distribution, and reproduction in any medium, provided the original work is properly cited.

In the article titled "Supervised and Unsupervised Subband Adaptive Denoising Frameworks with Polynomial Threshold Function" [1], there was an error in the Acknowledgments section, which should be corrected as follows:

"This work is fully supported by National Science and Technology Major Project of the Ministry of Science and Technology of China (no. 2015ZX03003010)."

\section{References}

[1] T. Gong, Z. Yang, G. Wang, and P. Jiao, "Supervised and unsupervised subband adaptive denoising frameworks with polynomial threshold function," Mathematical Problems in Engineering, Article ID 5203214, 12 pages, 2017. 


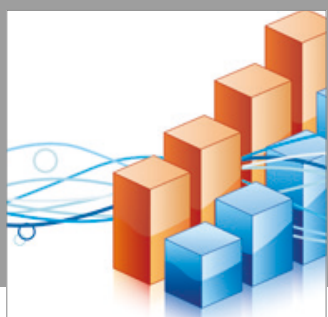

Advances in

Operations Research

vatersals

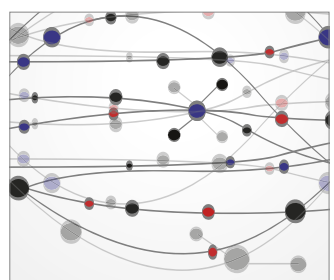

\section{The Scientific} World Journal
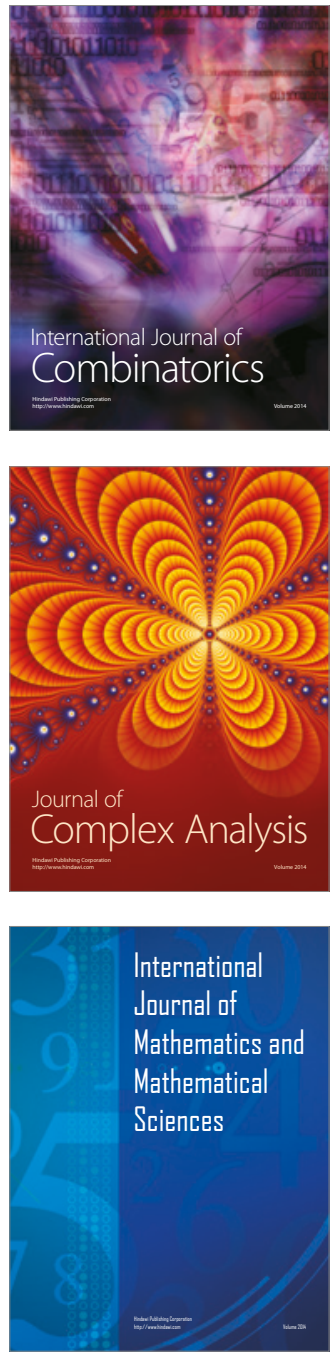
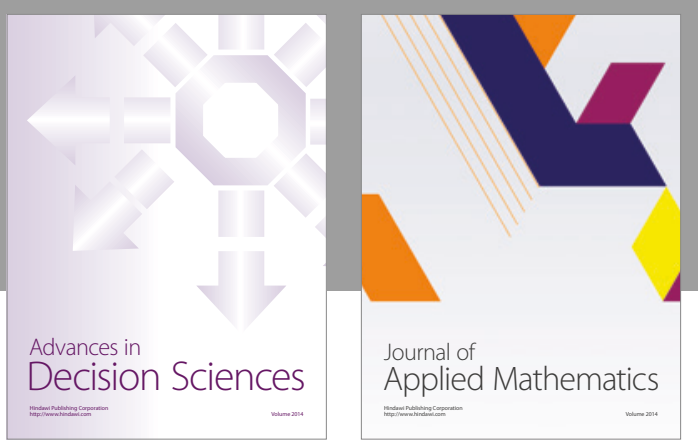

Algebra

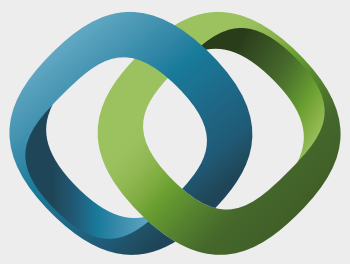

\section{Hindawi}

Submit your manuscripts at

https://www.hindawi.com
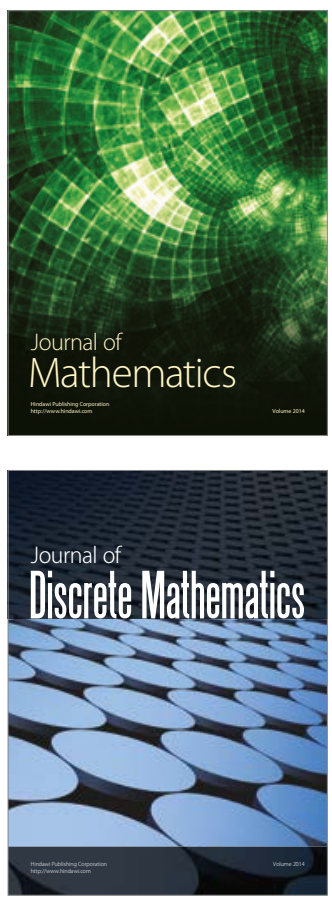

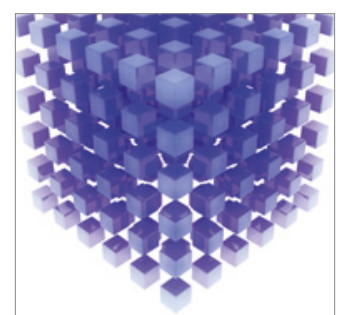

Mathematical Problems in Engineering
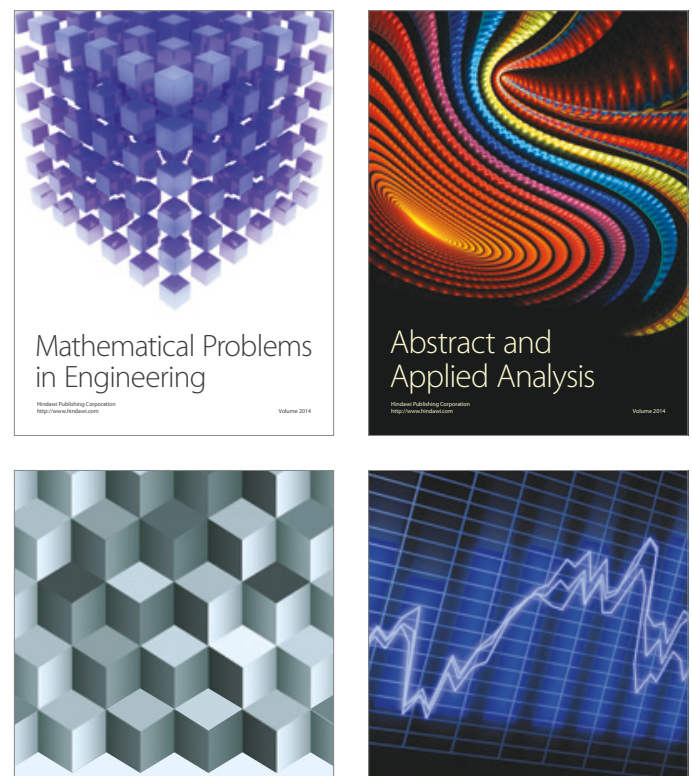

Journal of

Function Spaces

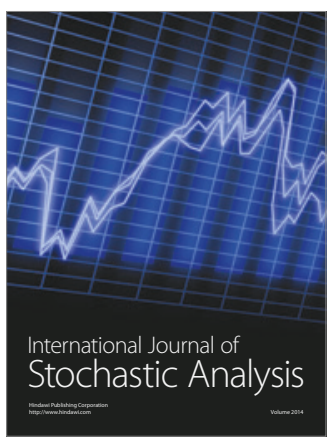

Probability and Statistics
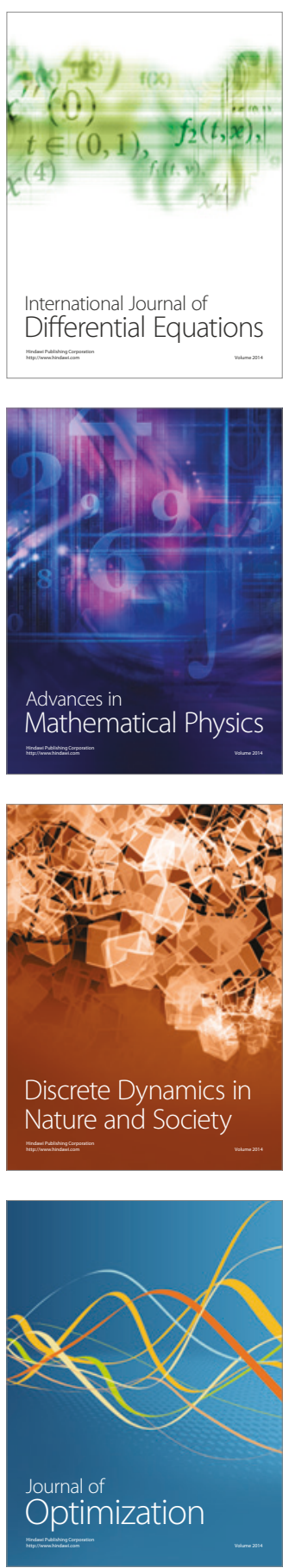